DOI: $10.2507 / 30$ th.daaam.proceedings. 010

\title{
THE USE OF OPTICAL SCANNER FOR ANALYSIS OF SURFACE DEFECTS
}

\author{
Michal Wieczorowski*, Bartosz Gapiński \& Natalia Swojak
}
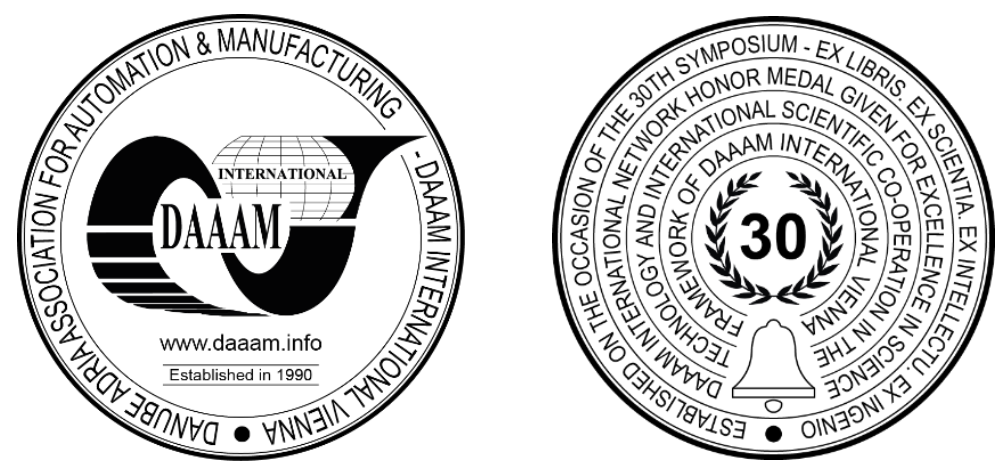

This Publication has to be referred as: Tatarevic, M[ichal]; Gapinski, B[artosz] \& Swojak, N[atalia] (2019). The Use of Optical Scanner for Analysis of Surface Defects, Proceedings of the 30th DAAAM International Symposium, pp.00760085, B. Katalinic (Ed.), Published by DAAAM International, ISBN 978-3-902734-22-8, ISSN 1726-9679, Vienna, Austria

DOI: $10.2507 / 30$ th.daaam.proceedings.010

\begin{abstract}
In the paper analysis of surface defect with a use of optical scanners was presented. Traditionally surface features on the top of surface layers is investigated by means of topography measurements in micro scale. In this case, asperities were much bigger, after surface treatment with aggressive media, and reaching as much as 1 millimetre. Due to this, analysis was conducted with optical scanner (using structural light or laser beam) and followed by appropriate data transmission, filtration and calculation procedures implemented in topography software. It proved that all important defects on the surface were properly detected. Dimensions of defects could be also inspected in all directions, as well as volume features and fractal dimension.
\end{abstract}

Keywords: surface defect; topography; optical scanner; mesoscale; surface treatment.

\section{Introduction}

The surface plays a pivotal role in many aspects of mechanical engineering and other areas. Its condition and the roughness present on it have a dramatic impact on a range of qualities, including contact mechanics or characteristics related, for instance, to friction and wearing out. The surface is a boundary between a solid body and the surrounding environment, for example, another object, substance or space, which makes it an element of the machine having direct and functional contact with the outside world.

The surface properties are directly linked to the damages of the parts of machines, as demonstrated, among others, by Tonshoff and Brinksmeier's works [1]. Specific surface characteristics also influence proper working of the parts of the machines. The surface is characterised by a specific system of geometric elements, which is called surface geometric structure (SGS). It comprises roughness, waviness, shape errors, and surface imperfections. This term clearly distinguishes roughness analysis from the description of defects, which are also treated in a completely different way in normative documents, though in some areas the understanding is different [2]. The surface roughness is treated as an outcome of a treatment process and parametrically characterised with numbers. Defects, on the other hand, result more from utilization processes and properties of the material [3] and in this case their assessment is arbitrary - in good/poor condition. Therefore, in general they can be desirable or undesirable, depending purely on the application or function of the surface and specified by e.g. length, depth, width, height or number per unit area. Nevertheless, defects description 
and characteristic are crucial in terms of surface qualification and the assessment of its quality. To distinguish defects on the surface from more general meaning of qualitatively defective work pieces, elements on the surface causing differences in appearance are called sometimes imperfections. Still, for the purpose of the research, the two terms are considered synonyms. According to [4] surface imperfection can be considered as element, irregularity or group of elements and irregularities of the real surface unintentionally or accidentally caused during manufacture, storage or use of the surface. As it was mentioned before, they are not related to surface roughness or waviness. Still they are assessed from reference surface which is related to the size of a single imperfection, suppressing the influence of form deviation on the assessment. There can be a lot of different surface imperfections, e.g. pores, blowholes, shrinkage holes, dents, warts, blisters, craters, as well as signs of erosion or corrosion.

Figure 1 presents an image of the workpiece with an imperfection and its scan in the meso-scale.

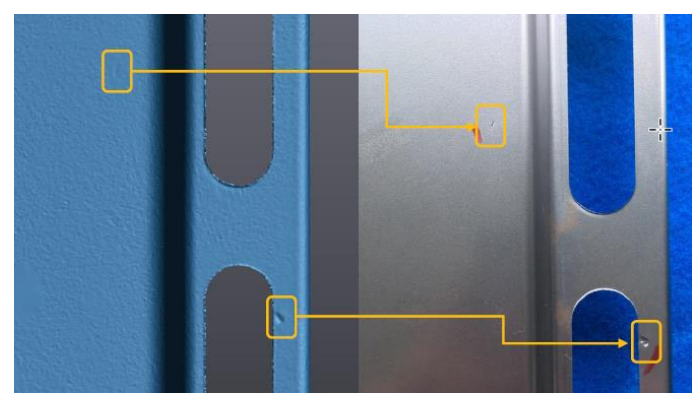

Fig. 1. Workpiece with an imperfection and its image taken with blue light laser scanner

Imperfections may be analysed in different scales, depending on their horizontal dimensions and depth (vertical dimensions). Micro-scale phenomena need to be measured with measurement devices of much finer resolution, whereas larger changes enforce a larger measurement range, often correlated with worsening of the resolution. In a large scale (macro), the length and angle metrology regards the measurements of geometric qualities, in the small scale (nano- and micro-) the surface roughness, and in the intermediate scale (meso) to the results obtained when roughness starts to transfer into geometric elements [5]. Traditionally, coordinate devices, among others, measuring machines and scanners were used in a macro-scale analyses, while in micro-scale e.g. profilometers and interferometers.

Meso-scale analyses, on the other hand, were performed with the use of the devices for shape errors measurement. There have been tendencies in recent years towards widening the ranges of some of the mentioned measuring devices, which resulted in a shift of the range within which they can be applied. Constructions aspiring to operate in meso- or even micro-scale are becoming to appear amongst coordinate measurement machines. White light profilometers and inferometers operating within a large range allow to measure not only the surface roughness, but also geometric qualities within the range of several dozen of millimetres.

The aim of the study was to investigate possibility of applying an optical scanner to inspect imperfections on the surface. When these features are in meso scale measuring them with a profilometer or a microscope (focus variation, confocal etc.) is very time consuming. Using optical scanner is much faster and allows for investigation of much bigger area.

\section{Measurements of the imperfections in the micro-scale}

There is an array of techniques allowing to reproduce the pattern of surface roughness in the micro-scale perceived as changes within the range not exceeding several dozen of micrometres. Within this range, they are analysed in connection with the surface roughness or as a function of their numerical characteristics. This is particularly relevant to corrosion phenomena. Sannareddy et al. used surface topography to analyse friction corrosion arising from lubricant deficiency during rolling [6]. Tomlinson and Farrell analysed roughness of chemically treated, e.g. through oxidation or hot-salt corrosion, surfaces of ceramic material [7].

Topographic analysis of the surface can be used to verify its corrosion resistance. Fig. 2a shows the picture of the topography of a surface, on which deep valleys occur and because of them a tendency to corrosion takes place. Fig. $2 \mathrm{~b}$ shows a different surface, with a height distribution close to normal on which this problem does not occur.

The most common technique to measure surface topography in micro scale is stylus profilometry. Commonly accepted, it has a significant drawback in touching a surface. From this point of view it is slow (limited traverse speed) and can damage some of the surface features. Optical techniques that substitute it more and more often are e.g. confocal profilometry [9], confocal microscopy [10], focus variation microscopy FVM [11] and coherence scanning interferometry CSI [12]. Some of them - particularly the two later ones - may also be used in meso-scale. Because of this, a large range scanning coherence interferometer was used as a reference for our measurements. 

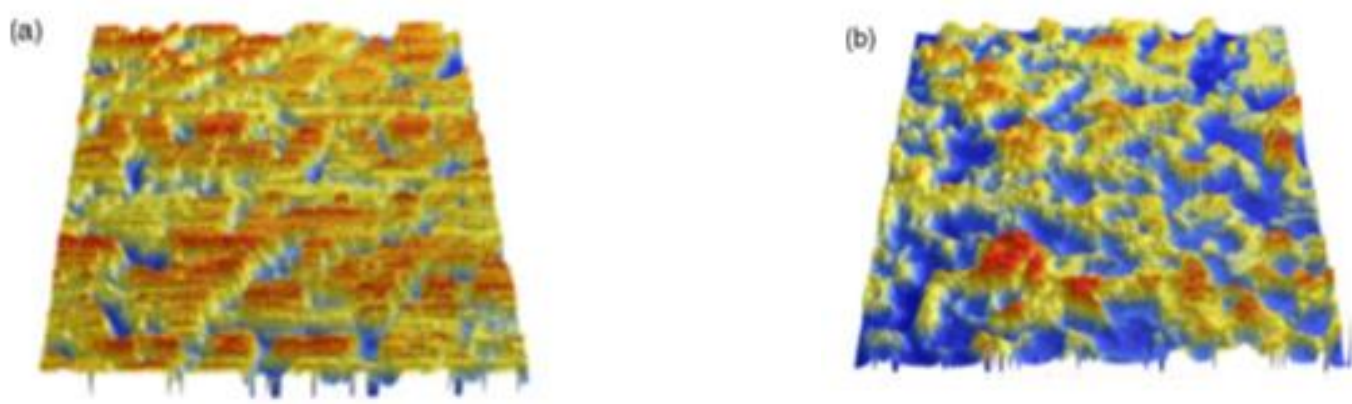

Fig. 2. Image of surface with tendency to corrosion (a), without tendency to corrosion (b) [8]

White light is commonly used in modern CSI systems. One of its more important advantages in comparison with monochromatic light is the fact that the latter does not allow to distinguish surface discontinuity, such as e.g. deep faults or high bands. If a slope is very steep, the device does not gather from it any measurement points, the data obtained are only for the peaks and valleys. In such a case, monochromatic light provides the result in the form of an image with lines at the top and at the bottom, without any information on their distance and thus does not allow to assess the height of roughness irregularities.

When white light is used, the bands obtained are of different colours. A separate set of bands arises simultaneously for each wavelength that forms white light and the intensity at every point of the image plane is the sum of bands' sets. This enables an analysis of significantly higher steps. When the difference of optical ways of the wave front returning from reference surface and the studied one is set for zero in the middle of the field, the interference bands coming from white light will appear in the image, coming from all wavelengths and having in this location their maximum intensities.

Due to the fact that the coherence length of white light usually equals a few wavelengths, the bands' system is different from the one in the case of monochromatic light. Two aspects of white light interferometry render it especially useful for the analysis of the surface qualities. Firstly, the interference occurs only when the difference of optical ways is approximate to zero, reducing in this way the ambiguity regarding location. Secondly, by contrast, the measurement range of the white light inferometer is limited only by the range of vertical scanning of mechanical system that moves the reference mirror.

\section{Imperfection measurements in the meso-scale}

Surface imperfections appear also in a bigger scale, on the limit of a range of many devices that are suitable for micro scale. In such a situation - apart from CSI and FVM - it is also possible to use optical scanners, working on triangulation principle. Their application for roughness or defect analysis was already investigation for concrete surfaces [13]. Their mechanism of action consists in analysing the light reflected or diffused by the surface of the element under study. It is possible to use structured light scanners or laser ones. The scanners which operate using structured light are usually mounted on stands or industrial robots' arms.

Systems basing on structured light projection use code patterns projections of structured light, e.g. Grey's codes for the element measured. This allows to create a digital reflection of the element under study basing on reading changes of the values (grey shadings) of each pixel and its surroundings. Subsequently, curves describing transitions from light to dark colour are made. Inflection points on these curves represent the edges. Currently, the most often adopted are hybrid methods which use the method of a bands' phase shift and Grey's code. This enables us to obtain very high measurement accuracy as well as high resolution [15].

Structured light scanners are more and more frequently replaced with laser scanners whose lower size ensures higher mobility and definitely lower sensitivity to external light. Initially, they were known as scanning laser heads or triangulation laser heads installed on coordinate machines or measuring arms. Recently they have been made to function as separate laser scanners, which may be operated in a manual or automatic mode, and have become one of the most common and effective methods of data acquisition.

The most frequent configuration of scanners basing on the structured light is as follows (fig. 3):

- 1 projector +1 camera (a)

- 1 projector +2 cameras (b) 
a)

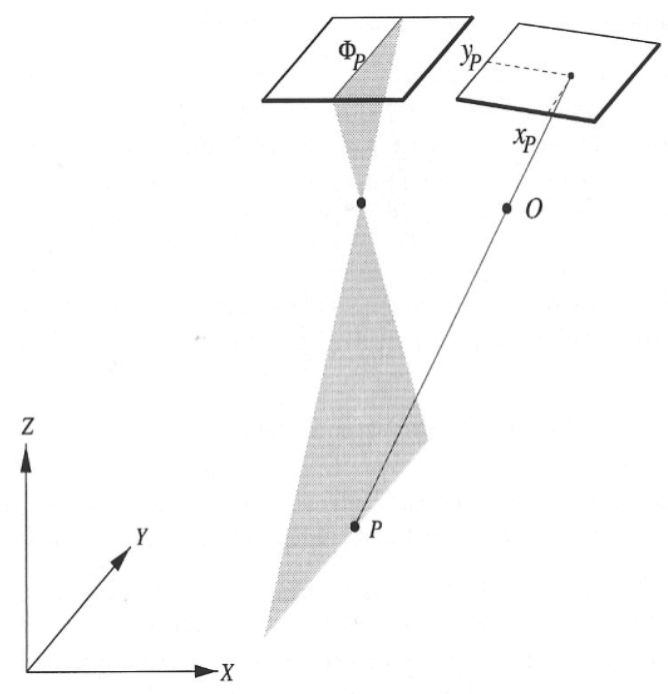

b)

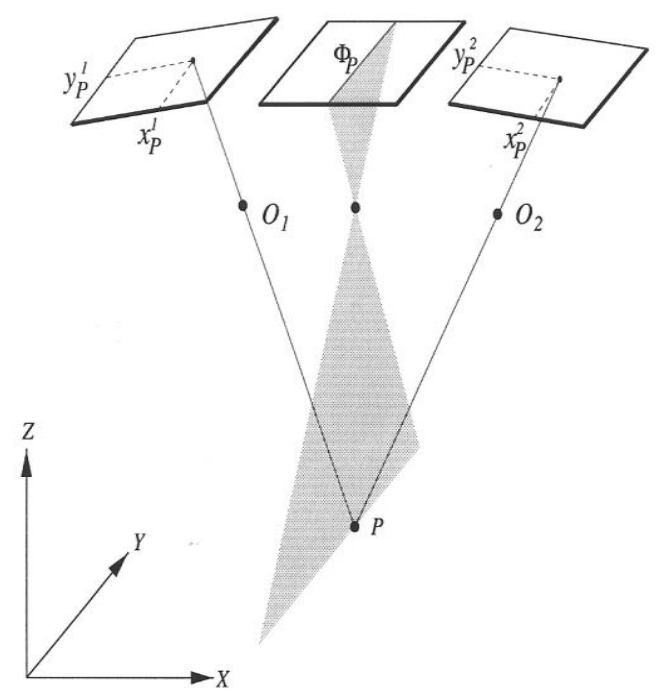

Fig. 3. The most common configurations of structured light scanners [14]

The operating principle of scanning heads is based on emitting, by the optical system, on the surface of the scanned object a point, line or a set of laser lines (up to 11 crosses) observed by a CCD camera equipped with a constant focal plane placed in a fixed distance from the laser (Fig. 4). Adopting a triangulation method and knowing the dependence of the transmitter and the camera, it is possible to determine the coordinates of the measurement points [16], [17]. It is possible to obtain a complete scan of the object thanks to the head movement along this object.

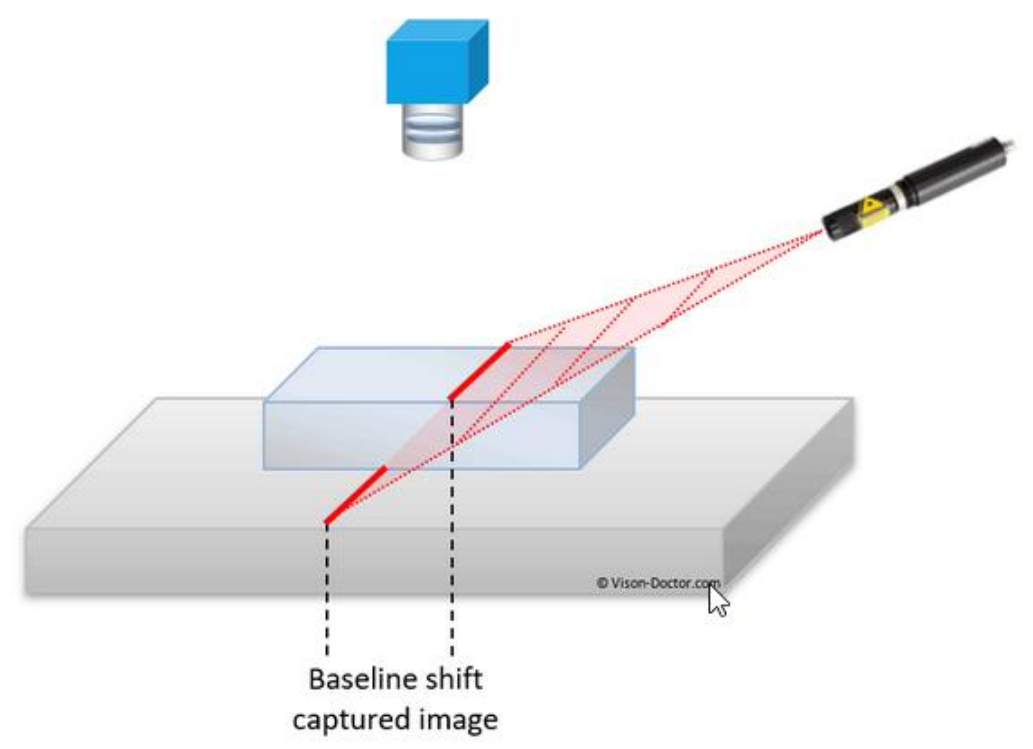

Fig. 4. The operating principle of a triangulation laser head [18]

In the case of scanning laser heads the relocating position in respect to the element scanned is determined with the data from the main axes of the measuring coordinative machine or the measuring arm. This means that the parts' reference system is closely connected with the device used for the completion of the specific task. A considerable difficulty arises when the application requires obtaining new data from the whole object in a single coordinate system. In such cases specific sides of the scanned object should be joined using their geometric qualities. This prolongs the scanning process and, at the same time, introduces an additional error into data acquisition. Another solution is to measure geometric qualities in separate measurement sessions, which excludes the possibility of determining their mutual relations.

Traditional scanners are used for the measurement of geometric elements [19], [20]. Using the scanners in the mesoscale has its limitations, yet an imperfections' analysis presented below demonstrates such a possibility. A comparison of performance of structured light and laser scanner was performed in macro scale [21], but not for surface inspection. 


\section{Measurement setup and procedure}

Elements with surface imperfections following mechanical and chemical trials, including corrosion in a strong aggressive environment were analysed in the study. Specific samples were collected for each element and 10 measurement regions were identified. For each sample parametric analysis was performed. Every surfaces were inspected with a scanner or coherence scanning interferometer. From the raw set of data a measurement region was then chosen and coordinates of measurement points were extracted. This region obtained from a scanner is usually much bigger than the one obtained from a CSI. Data points from scanner were optimized [22] and interpolated to get a more dense set of coordinates. The set was subsequently transformed to a surface topography software (Mountains Map) for further evaluation. Data with form and inclination removed using a Gaussian filter were used to calculate topography parameters, according to [23]. These parameters were:

- maximum height of the scale-limited surface $S z$, considered as a sum of the maximum peak height value and the maximum pit height value,

- arithmetical mean height of the scale limited surface $S a$, i.e. arithmetic mean of the absolute of the ordinate values within a definition area,

- reduced peak height Spk which is equal to average height of the protruding peaks above the core surface,

- core height Sk considered as a distance between the highest and lowest level of the core surface,

- reduced dale height Svk i.e. average height of the protruding dales below the core surface,

- material ratio Smr1 which is peaks ratio of the area of the material at the intersection line separating protruding hills from the core surface to the evaluation area,

- material ratio Smr2 equal to dales ratio of the area of the material at the intersection line separating protruding dales from the core surface to the evaluation area.

High range coherence scanning interferometer TMS 500 was used as a reference, thanks to its good resolution, high vertical range and possibility of inspecting area of 30x35 mm in one scanning cycle. Furthermore, the surfaces were inspected by means of structured white light scanner ATOS and blue light laser scanner HandySCAN 3D.

\section{Results and discussion}

Examples of images of surfaces as well as measurement results were presented below. Figure 5 shows a surface obtained by means of a CSI.
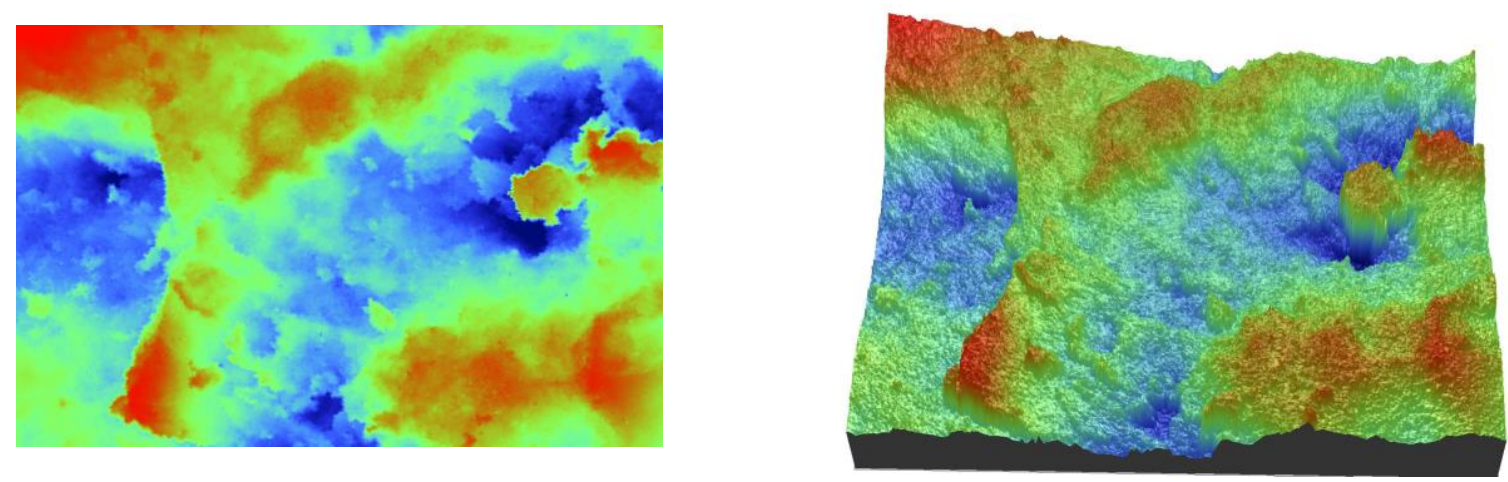

Fig. 5. Image of the corroded surface obtained with a high range coherence scanning interferometer

For this surface the following reference values of parameters were obtained as an average of 3 measurements:

\begin{tabular}{|c|c|}
\hline Parameter & Value $[\boldsymbol{\mu m}]$ \\
\hline $\mathrm{Sz}$ & 0,593 \\
\hline $\mathrm{Sa}$ & 0,077 \\
\hline $\mathrm{Spk}$ & 0,078 \\
\hline $\mathrm{Sk}$ & 0,103 \\
\hline $\mathrm{Svk}$ & 0,425 \\
\hline $\mathrm{Smr} 1$ & $9,001 \%$ \\
\hline $\mathrm{Smr} 2$ & $86,301 \%$ \\
\hline
\end{tabular}

Table 1. Obtained parameters value 
The results for series of 5 measurements performed with a structured light scanner were presented as plots on figure 6 .

\section{Structured}

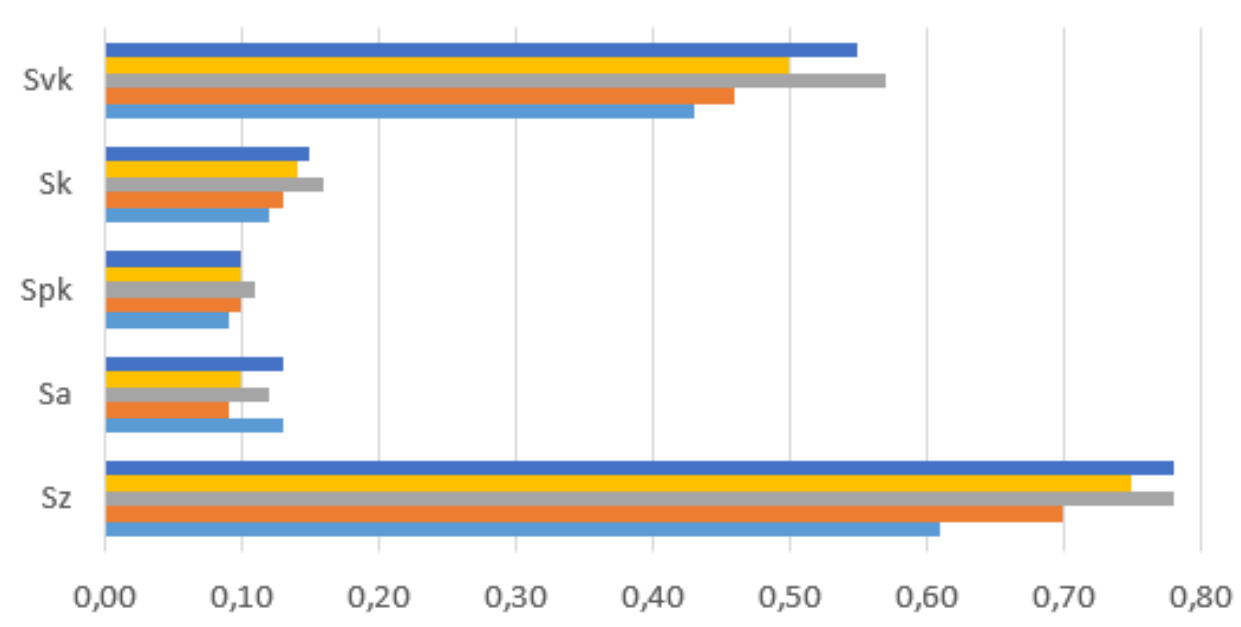

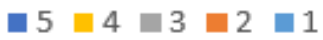

Smr1 Structured

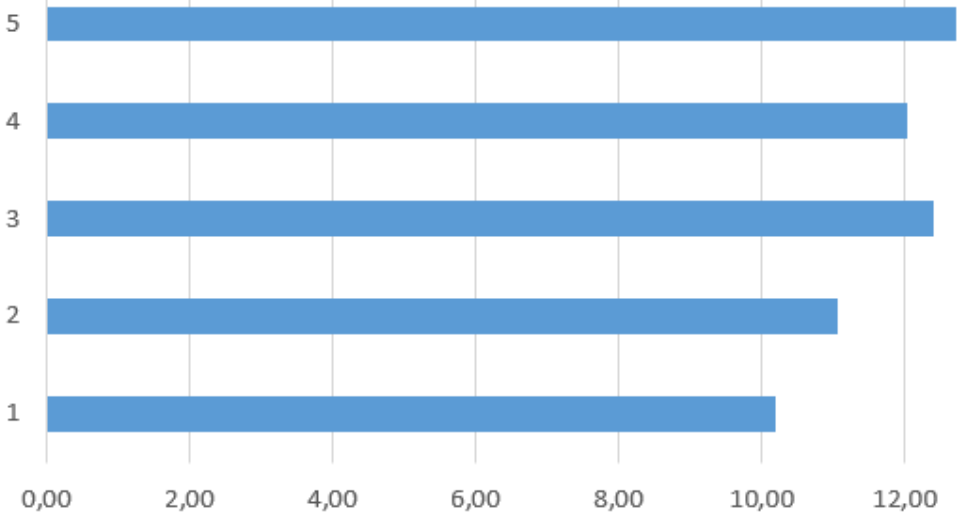

Smr2 Structured

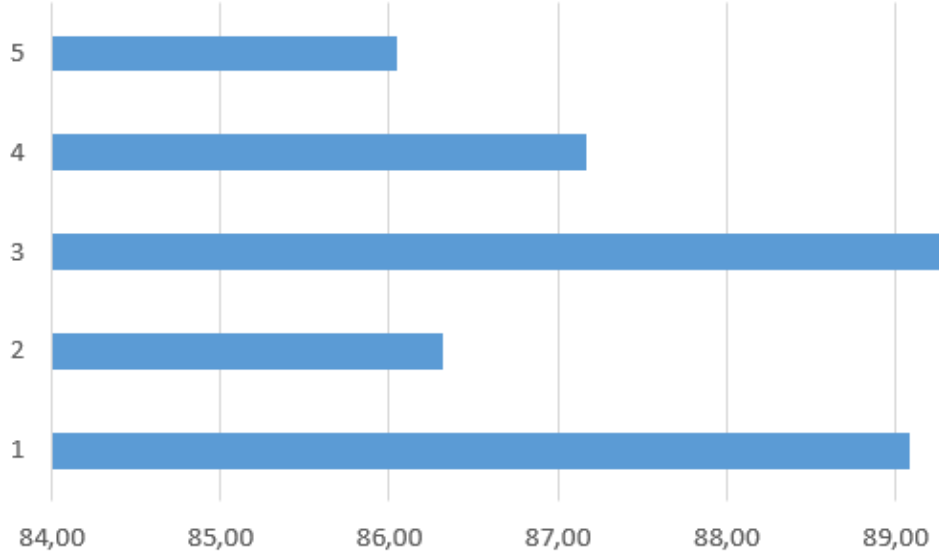

Fig. 6. Measurement results obtained with a structured light scanner

The plots show quite good repeatability. Values of ranges for particular parameters are: 0,17 for Sz, 0,04 for Sa, 0,02 for Spk 0,04 for Sk, 0,14 for Svk and 2,54 for Smr1 and 3,22 for Smr2 respectively. This repeatability is not much worse 
than the values obtained with many traditional roughness measurements. The next picture (fig. 7) show the results for series of 5 measurements performed with a laser scanner.

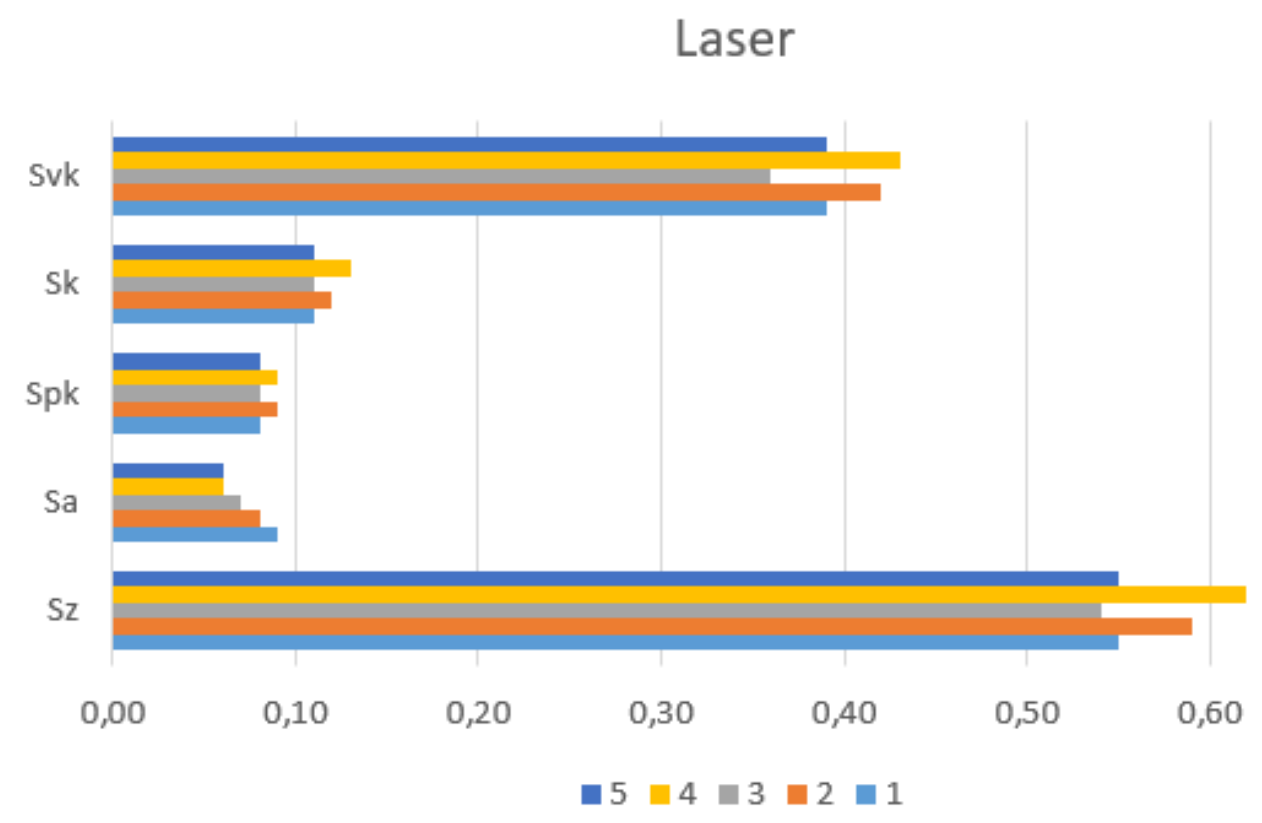

\section{Smr1 Laser}

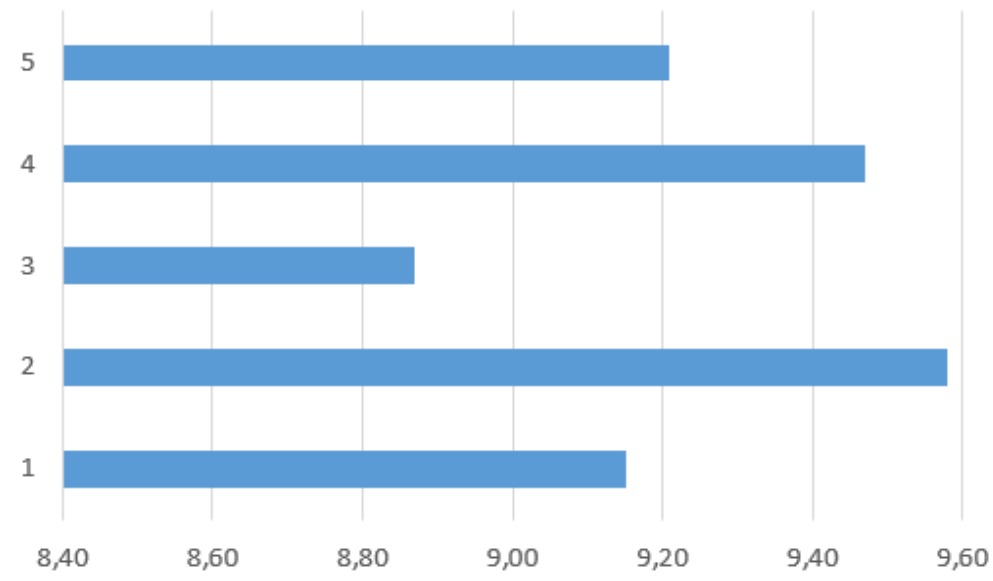

Smr2 Laser

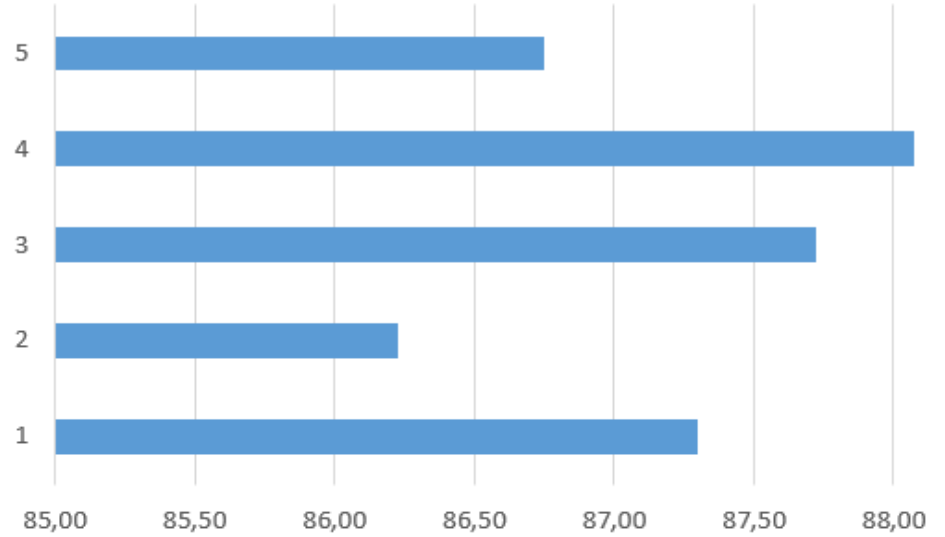

Fig. 7. Measurement results obtained with a laser scanner

Here the plots show even better repeatability. Values of ranges for particular parameters are: 0,08 for Sz, 0,03 for Sa, 0,01 for Spk 0,02 for Sk, 0,07 for Svk and 0,71 for Smr1 and 1,84 for Smr2 respectively. This repeatability, in most cases 
better than $20 \%$ in relation to mean value of parameter is similar to the values obtained with traditional and optical roughness measurements. Moreover, the repeatability data for laser scanner are approximately twice better than the ones obtained for a structured light scanner. On figure 8 comparison of mean values obtained for both scanners and coherence scanning interferometer was shown.

\section{Comparison}

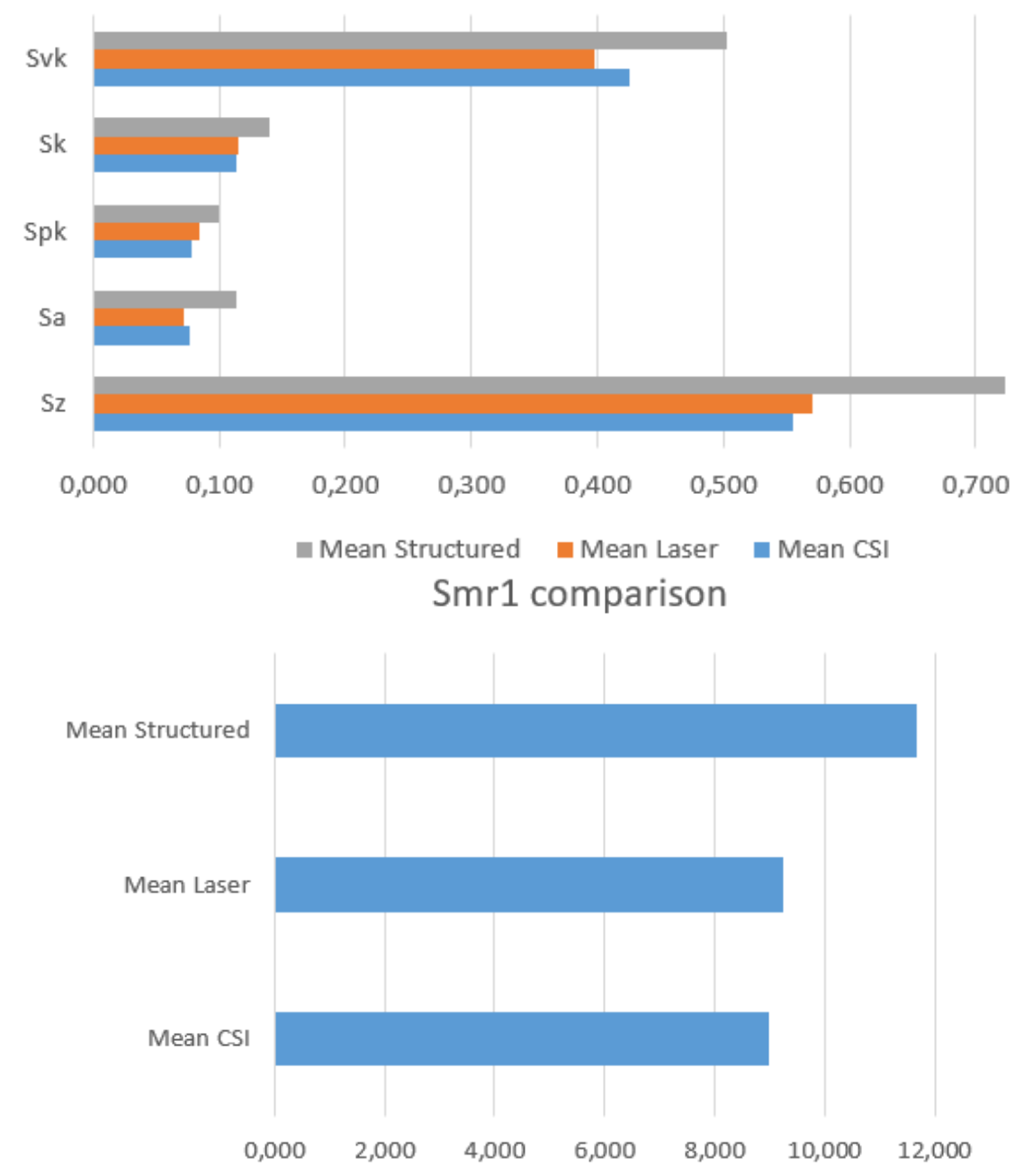

\section{Smr2 comparison}

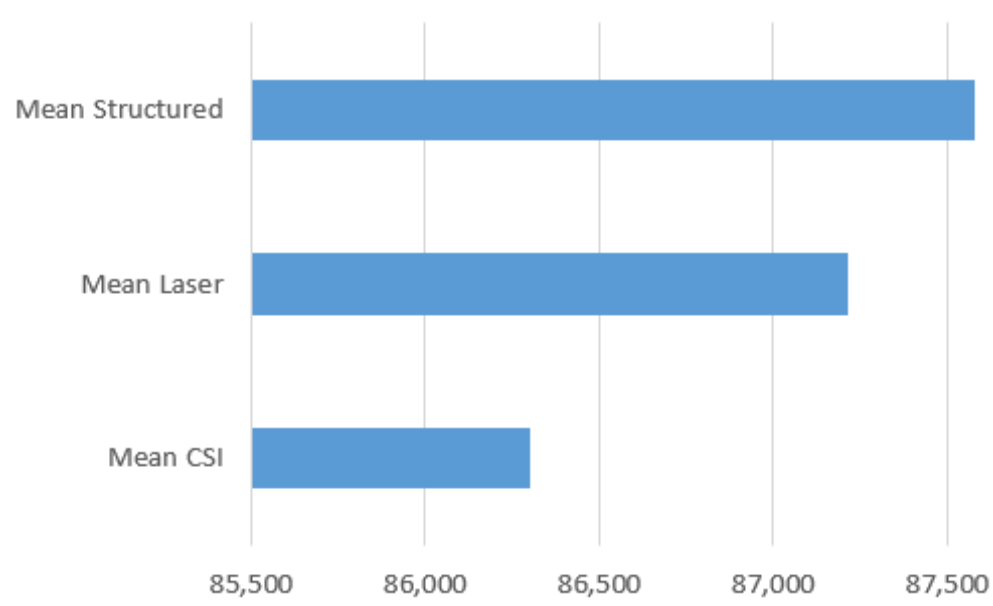

Fig. 8. Comparison of measurements performed with a CSI and scanners 
The comparison shows good agreement between laser scanner measurement results and the ones obtained with a reference device (CSI). The differences in relation to the values of parameters are less than $10 \%$. For structured light scanner the results were a little worse and differences in relation to the values in relation to the values only in some cases were smaller than $20 \%$.

\section{Conclusions}

In the paper the use of optical scanners to detect and measure surface imperfections or defects was presented. This method can be used in meso scale, when vertical and horizontal resolution is not as good as the one that is typical for surface topography measuring instruments in micro scale. Application of optical scanners for this task is new, but shows quite good possibilities. Data required special preparation and using a software that is commonly applied for inspection of asperities.

Data obtained for laser scanner were significantly better than the ones obtained for a structured light scanner. This shows advantage of new emerging technology, as the structured white light scanner was using technology which is not the newest one. On the contrary technology used in a blue light scanner is at present the most up to date. Also results obtained with laser scanner showed very good agreement with the ones obtained with a coherence scanning interferometer.

In this research possibility of applying an optical scanner to inspect imperfections on the surface was investigated. The assumption was that optical scanner is much faster than a profilometer or a microscope and allows for investigation of much bigger area, in cases when its resolution is good enough to represent the surface imperfections properly. The results showed that the results are promising for all the defects falling into meso scale.

The technique of optical scanning for that application has some limitations. The most important one is resolution of a measurement system, which regards to both: vertical and horizontal ones. If the imperfections are too small, a scanner will most probably not detect it or distort it, basing on very few points only. Still, as it was proved in the paper, particularly a laser scanner is a perfect solution when inspection of big areas with moderate resolution is concerned. All other techniques are too time consuming.

Future works in this area will comprise elaboration of a more sophisticated algorithm to combine points obtained in different scales, that will enable for collecting a set of data with a scanner and partially, in most crucial regions, adding points using a better resolution technique. It is also planned to develop this ideas with computed tomography.

\section{Acknowledgments}

The presented research results were supported by the Polish National Centre of Research and Development (project contract No. Innotech In-Tech K2/IN2/58/182896/NCBR/12; "Elaboration of manufacturing technology of new generation ultralight seats for public transportation fulfilling requirements of UE directives, UN regulations and American White Book") and PUT project 02/22/SBAD/1501.

\section{References}

[1] Tönshoff H.K., Brinksmeier E. (1980). Determination of the mechanical and thermal influences on machined surfaces by microhardness and residual stress analysis, Ann.CIRP, 29, 519-530

[2] Brcic M., Canadija M., Brnic J. (2016). Influence of imperfections on carbon nanotube properties, 8nd International Conference on Physical and Numerical Simulation of Materials Processing, ICPNS'16, Seattle, Washington, USA, pp. 1-6

[3] Wojciechowski L., Wieczorowski M., Mathia T.G. (2017). Transition from the boundary lubrication to scuffing the role of metallic surfaces morphology, Wear, 392-393, 39-49

[4] ISO 8785:1998

[5] Gapiński B., Wieczorowski M., Marciniak-Podsadna L., Swojak N., Mendak M., Kucharski D., Szelewski M., Krawczyk A. (2019). Use of White Light and Laser 3D Scanners for Measurement of Mesoscale Surface Asperities, rozdział w: Advances in Manufacturing II, V.5, Metrology and Measurement Systems, ed. Diering M., Wieczorowski M., Brown C.A., Springer, 239-256

[6] Sannareddy H., Raja J., Chen K. (1998). Characterization of surface texture generated by multi-process manufacture, International Journal of Machine Tools and Manufacture, 38, 5-6, 529-536

[7] Tomlinson W.J., Farrell J. (1992). Fracture strength, surface roughness and corrosion of ceramics, Cer.Int., 18, 355-357

[8] Mathia T.G., Pawlus P., Wieczorowski M. (2011). Recent trends in surface metrology, Wear, 271, 3-4, 494-508

[9] Butler D.J., Horsfall A., Hrynevych M., Kearney P.D., Nugent K.A. (1993). Confocal profilometer with nanometric vertical resolution, Optics Communications, 100, 1-4, 87-92

[10] Sheppard C.J.R., Matthews H.J. (1998). The extended focus, auto-focus and surface profiling techniques of confocal microscopy, Journal of Modern Optics, 35, 1, 145-154

[11] Subbarao M., Choi T. (1995). Accurate Recovery of Three-Dimensional Shape from Image Focus, IEEE Trans. on Pattern Analysis and Machine Intelligence, 17, 266-274 
[12] Deck L., de Groot P. (1995). High-speed non-contact profiler based on scanning white light interferometry, International Journal of Machine Tools and Manufacturing, 35, 147-150

[13] Majchrowski R., Grzelka M., Wieczorowski M., Sadowski L., Gapiński B., (2015). Large area concrete surface topography measurements using optical 3D scanner, Metrology and Measurement Systems, XXII, 4, 565-576

[14] Luhmann T., Robson S., Kyle S., Harley I. (2011). Close range photogrammetry. Principles, techniques and applications, Whittles Publishing, Dunbeath

[15] Geng J. (2011) Structured-light 3D surface imaging: a tutorial, Optical Society of America

[16] Park H.S., Tuladhar U.M. (2014). Development of an Inspection System for Defect Detection in Pressed Parts using Laser Scanned Data, Procedia Engineering, 69, 931-936

[17] Park H.S., Tuladhar U.M. (2014). Development of a Quality Inspection System Using Laser Based Scanner, Chapter 27 in DAAAM International Scientific Book 2014, pp. 339-356, B. Katalinic (Ed.), DAAAM International, Vienna, Austria

[18] https://www.vision-doctor.com/en/laser-illumination/principle-of-triangulation.html, September, 2019

[19] Gapinski B., Wieczorowski M., Marciniak-Podsadna L., Dybala B., Ziolkowski G. (2014). Comparison of Different Methods of Measurement Geometry Using CMM, Optical Scanner and Computed Tomography 3D, Procedia Engineering, 69, 255-262

[20] Tomasiak J. (2014). The use of Optical Methods for Leak Testing Dampers, Procedia Engineering, 69, 788-794

[21] Tóth T., Živčák J. (2014). A Comparison of the Outputs of 3D Scanners, Procedia Engineering, 69, 393-401

[22] Cupar A, Pogacar V., Stjepanovic Z. (2013). Methodology For Analysing Digitised Geometry, Chapter 56 in DAAAM International Scientific Book 2013, pp. 903-920, B. Katalinic \& Z. Tekic (Eds.), DAAAM International, Vienna, Austria

[23] ISO 25178-2:2012. 